\title{
Targeting TRP channels: beyond TRPV1
}

\author{
Arthur Gomtsyan • Arpad Szallasi
}

Received: 9 January 2015 / Accepted: 12 January 2015 /Published online: 10 February 2015

(C) Springer-Verlag Berlin Heidelberg 2015

This theme issue of Naunyn-Schmiedeberg's Archives of Pharmacology contains six review articles covering recent advances in our understanding of the potential pharmacological roles of different transient receptor potential (TRP) ion channel subtypes that can be targeted for the therapeutic use. Numerous review articles and several book chapters in recent years have extensively covered the advances in modulation of TRPV1, founding member of TRP family. The intended purpose of this theme issue was to summarize the knowledge about other less studied TRP subtypes. For comparison, as of early January 2015, SciFinder identifies 8814 publications for TRPV1, but only 2410 for TRPA1, 1759 for TRPV4, and 1608 publications for TRPM8.

Transient receptor potential melastatin 8 (TRPM8) can be activated by cold temperatures $\left(8-25^{\circ} \mathrm{C}\right)$ and cooling agents such as menthol and icilin. Several studies with TRPM8 knockout mice demonstrated significant deficiencies to cold responses including cold allodynia. Additionally, there are reports demonstrating strong association of the receptor with migraine. Besides, TRPM8 was considered also as a potential therapeutic target for chronic pain. However, pharmacological studies with potent TRPM8 antagonist AMG2850 (Lehto et al. 2015) revealed that despite significant antagonism demonstrated in in vivo target engagement models, AMG2850 showed no efficacy in the animal models of inflammatory

\footnotetext{
A. Gomtsyan $(\bowtie)$

Department of Chemistry, Global Pharmaceutical Research and Development, AbbVie, North Chicago, IL 60064, USA

e-mail: Arthur.r.gomtsyan@abbvie.com

A. Szallasi $(\bowtie)$

Department of Laboratories, Monmouth Medical Center, Long

Branch, NJ 07740, USA

e-mail: ASzallasi@barnabashealth.org
}

and neuropathic pain. Authors suggest that either TRPM8 does not play a role in mechanical pain behaviors measured or not high enough target coverage was achieved in these experiments.

In most species (including humans), transient receptor potential ankyrin 1 (TRPA1) mainly functions as a chemosensor as it can be activated by a variety of pungent chemicals found in spices such as allicin and diallyl disulfide (garlic), cinnamaldehyde (cinnamon) and isothiocyanates (horseradish, wasabi, mustard oil), as well as by environmental irritants found, for example, in cigarette smoke and exhaust fumes. By contrast, TRPA1 seems to play various roles in temperature detection in different species: for example, it detects heat in insects, it responds to noxious cold in rodents, and it is temperature-insensitive in nematodes. This diversity hinders the extrapolation of results obtained in preclinical models employing temperature challenge to humans. The thermosensitivity of human TRPA1 remains debated, though most studies imply (at least under pathological conditions such as nerve injury) a cold thermosensor. Because TRPA1 is expressed in both neuronal and non-neuronal tissues, the therapeutic potential of the receptor was studied in a number of disease models. Chen and Hackos (2015) summarize available data on the role of TRPA1 in pain relief, airway inflammation, and pruritus.

Two reviews cover the potential therapeutic utility of targeting TRPV4 channel for various musculoskeletal diseases as well as pulmonary diseases. TRPV4 as a sensor of mechanical and osmotic stimuli plays a key role in musculoskeletal tissues including bone, cartilage, and synovia (McNulty et al. 2015). The degree of TRPV4 activation is critical for joint health as TRPV4 gain of function mutations cause skeletal dysplasia while TRPV4-deficient mice develop osteoarthritis. Therefore, both TRPV4 agonists and 
antagonists may find therapeutic utility by establishing the balance of joint TRPV4 activity in pathological conditions.

Goldenberg et al. (2015) reviewed the role of TRPV4 in pulmonary homeostasis and diseases, such as pulmonary edema, pulmonary hypertension, and lung injury. Role of TRPV4 inhibitors for acute respiratory distress syndrome is rationalized in terms of TRPV4 actions in not only lung but also in immune cells. Authors also draw attention to potential safety issues of targeting TRPV4 due to multifunctional nature and wide expression pattern of the receptor.

Chronic itch is debilitating condition that is closely related to pain in that low intensity stimuli is eliciting itch and high intensity stimuli causing pain. Itch can be modulated through both central and peripheral mechanisms. Zhang (2015) discusses involvement of subset of TRP channels, TRPA1, TRPV1, TRPV3, TRPV4, and TRPM8, in itch by neuronal and non-neuronal mechanisms. Mechanistic understanding behind modulation of both histaminergic and nonhistaminergic itch by different TRPs is also reviewed.

Cough is the most common symptom of many respiratory diseases, but it can be a disorder by itself. Many TRPs are widely distributed throughout the respiratory tract modulating inflammation and responding to a variety of stimuli such as temperature, osmolarity, and oxidative stress. Bonvini et al. (2015) summarize the current knowledge on physiological role of TRPV1, TRPA1, TRPV4, and TRPM8 in cough and present data from available clinical experience with antagonists of these TRP's.

\section{References}

Bonvini SJ, Birrell MA, Smith JA, Belvisi MG (2015) Targeting TRP channels for chronic cough: from bench to bedside. Naunyn Schmiedebergs Arch Pharmacol. This issue

Chen J, Hackos DH (2015) TRPA1 as a drug target-promise and challenges. Naunyn Schmiedebergs Arch Pharmacol. This issue

Goldenberg NM, Ravindran K, Kuebler WM (2015) TRPV4: physiological role and therapeutic potential in respiratory diseases. Naunyn Schmiedebergs Arch Pharmacol. doi:10.1007/s00210-014-1058-1, This issue

Lehto SG, Weyer AD, Zhang M, Youngblood BD, Wang J, Wang W, Kerstein PC, Davis C, Wild KD, Stucky CL, Gavva NR (2015) AMG2850, a potent and selective TRPM8 antagonist is not effective in rat models of inflammatory and neuropathic pain. Naunyn Schmiedebergs Arch Pharmacol. This issue

McNulty AL, Leddy HA, Liedtke W, Guilak F (2015) TRPV4 as therapeutic target for joint diseases. Naunyn Schmiedebergs Arch Pharmacol. doi:10.1007/s00210-014-1078-x, This issue

Zhang X (2015) Targeting TRP ion channels for itch relief. Naunyn Schmiedebergs Arch Pharmacol. doi:10.1007/s00210-014-1068-Z, This issue 